\title{
GENETICS
}

\section{Etiological insight into the genetic association of TCPTP with RA and JIA?}

Genome-wide association studies have linked polymorphisms in the PTPN2 gene, encoding T-cell protein-tyrosine phosphatase (TCPTP), with susceptibility to rheumatoid arthritis (RA) and juvenile inflammatory arthritis (JIA). New data from Doody et al. provide insight into the mechanistic basis of these relationships.

Previously, this group demonstrated that Ptpn 2 knockout mice produce high levels of TNF-a cytokine central to RA pathogenesis-and have progressive systemic inflammation. Additionally, "we noted that the bones of Ptpn $2^{-/-}$mice were very brittle," claims Doody.

In light of these findings and the genetic association of TCPTP with RA, "we were eager to determine if Ptpn $2^{-/-}$mice had a similar bone and joint phenotype as seen in human RA," says Doody. Subsequent investigation revealed synovitis and severe resorption of subchondral bone in Ptpn $2^{-/-}$mice, accompanied by increased osteoclast abundance, and enrichment of inflammatory cells and cytokines in the joint, compared with littermate controls.

"The inflammation in the joints of $P t p n 2^{-/-}$mice was unique to that tissue when compared to the inflammation we have previously described in other tissues," states Doody. However, owing to systemic inflammation and premature death in $P t p n 2^{-/-}$mice, further studies are needed to investigate TCPTP in specific tissues, and in adult mice. "By enhancing our knockout mouse model with inducibility, reversibility, and tissue specificity, we will be able to better understand the functions of TCPTP", concludes Doody.

\section{David Killock}

Original article Doody, K. M. et al.T cell tyrosine phosphatase deficiency results in spontaneous synovitis and subchondral bone resorption in mice. Arthritis Rheum. doi:10.1002/art.33399 